\title{
Visualizing the Local Experience: HIV Data to Care Tool
}

\author{
Lauren E. Snyder*1, Dean McEwen1, Mark Thrun ${ }^{2,1}$ and Arthur Davidson ${ }^{1}$ \\ ${ }^{1}$ Public Health Informatics Group, Denver Public Health, Denver, CO, USA; ${ }^{2}$ Gilead, Denver, CO, USA
}

\section{Objective}

To describe Denver Public Health's model for designing a business intelligence (BI) tool for HIV surveillance and outreach and the impact after implementation.

\section{Introduction}

Recently signed in Denver, the Paris Declaration demonstrates a collective resolution to end AIDS by continually monitoring these goals. However siloed data and in/out migration results in poor capacity to track population level care indicators for persons living with HIV (PLWH). Surveillance should not only enumerate PLWH but also support prevention and care programming (1). We designed and implemented the HIV Data to Care Tool to describe the continuum, from case finding to HIV care. This study describes a system to combine data sources to inform local HIV surveillance, outreach, and care. Development objectives included targeted community and clinical interventions and evaluation, user defined reports to identify subpopulation disparities, and a persistent data visualization readily available to stakeholders.

\section{Methods}

Existing local data sources were integrated into a common data infrastructure, including: CDC's HIV surveillance system from Denver and 4 Denver-adjacent counties, US Census denominators and demographics, state mandated clinical measure reporting (viral load and CD4 counts), and HIV clinic encounter data (e.g., sexually transmitted infections, pharmacy and prevention measures). Denver Health, an integrated safety-net provider, serves $25 \%$ of Denver's residents with electronic health record data for its patients. Collaborative requirements gathering defined needs and specifications, including wireframes, a data dictionary, and business needs. The web-based, custom developed business intelligence dashboard was built with MS-SQL Server Reporting Services and deployed after testing for quality assurance and usability.

\section{Results}

Requirements gathering identified six population health use cases (and dashboards) for integrated data analysis, within a BI environment: Diagnosis Summary, Linkage to Care, Retention in Care, Care Summary, Community Viral Load, and a Surveillance Summary Page. Three patient line lists were also developed to meet outreach worker needs. Users control content through configurable parameters including demographics, location of PLWH residence, care site, and transmission category, allowing custom reporting to identify disparities (i.e., black MSM incidence rates), see Figure 1.

\section{Conclusions}

The tool integrated existing regional data sources and permitted new, near real-time visualization of complex information. Utilized locally for program planning and patient management, it provided a more nuanced understanding of the local epidemic and benefited resource allocation. It has improved evaluation, assessment and quality improvement of linkage,, reengagement, and clinical outcomes by subpopulations, including identification of those not in care. Additionally reporting has supplemented the Denver Community Health Assessment, grant applications, and strengthened regional realtionships through collaborative development. New efforts are focused on extending the tool to serve adjacent counties and integrating new data soruces for more comprehnshive reporting. A jurisdiction may benchmark an HIV-infected individual's or population's movement through the care continuum (2), gauge intervention effectiveness, and generate timely, user driven reports. The tool's development and implementation model have proven reusable and translatable to address other priorities (e.g., chronic disease and immunization coverage).

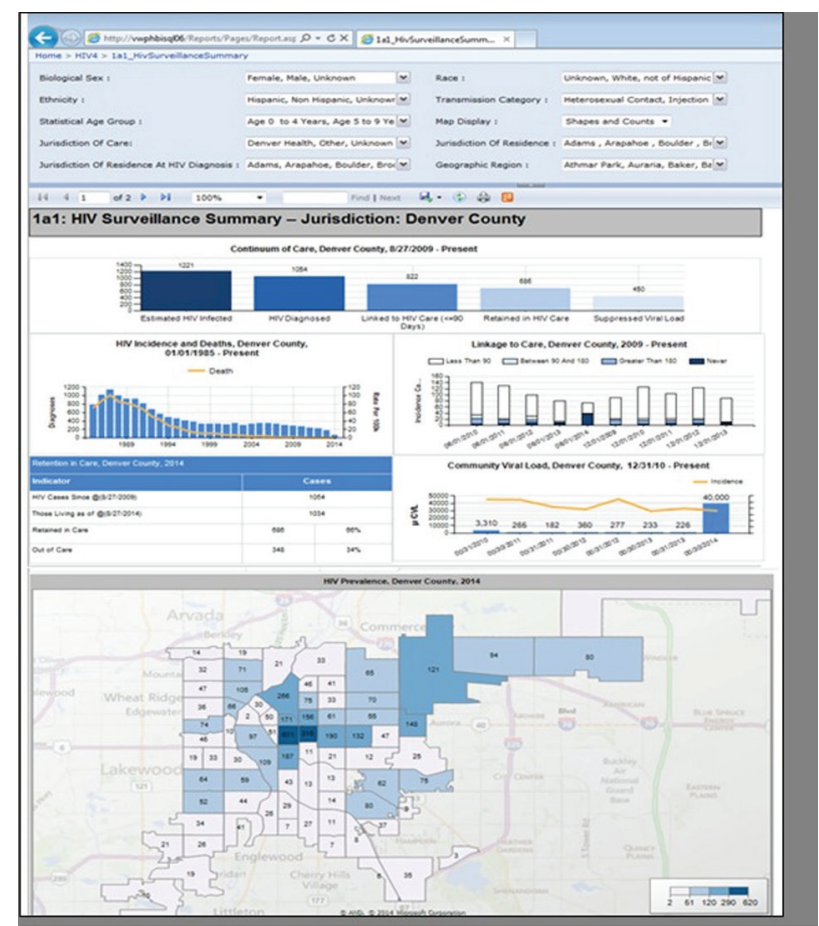

Figure 1. Screenshot of the Data to Care Tool: HIV Surveillance Summary Report Page

Figure 1: Screenshot of the HIV Data to Care Tool Surveillance Summary Page

Keywords

Informatics; Visualization; HIV

\section{References}

1. Sweeney P, Gardner L, Buchaczk, Garland P, Mugavero M, Bosshart J et al. Shifting the Paradigm: Using HIV Surveillance Data as a Foundation for Improving HIV Care and Preventing HIV Infection. Milbank Quarterly. 2013;91(3):558-603.

2. Gardner E, McLees M, Steiner J, del Rio C, Burman W. The Spectrum of Engagement in HIV Care and its Relevance to Test-and-Treat Strategies for Prevention of HIV Infection. Clinical Infectious Diseases. 2011;52(6):793-800.

\section{*Lauren E. Snyder}

E-mail: lauren.snyder@dhha.org 